\title{
Tailoring Dispersion properties of photonic crystal waveguides by topology
} optimization

\section{Stainko, Roman; Sigmund, Ole}

Published in:

Waves in Random and Complex Media

Link to article, DOI:

10.1080/17455030701501851

Publication date:

2007

Document Version

Early version, also known as pre-print

Link back to DTU Orbit

Citation (APA):

Stainko, R., \& Sigmund, O. (2007). Tailoring Dispersion properties of photonic crystal waveguides by topology optimization. Waves in Random and Complex Media, 17(4), 477-489.

https://doi.org/10.1080/17455030701501851

\section{General rights}

Copyright and moral rights for the publications made accessible in the public portal are retained by the authors and/or other copyright owners and it is a condition of accessing publications that users recognise and abide by the legal requirements associated with these rights.

- Users may download and print one copy of any publication from the public portal for the purpose of private study or research.

- You may not further distribute the material or use it for any profit-making activity or commercial gain

- You may freely distribute the URL identifying the publication in the public portal 


\title{
Tailoring dispersion properties of photonic crystal waveguides
}

\section{by topology optimization}

\author{
R. STAINKO $\dagger$ and O. SIGMUND* $\ddagger$
}

$\dagger$ Department of Mathematics, Matematiktorvet 303S,

$\ddagger$ Department of Mechanical Engineering, Nils Koppels Alle 404,

Technical University of Denmark, DK-2800 Kgs. Lyngby, Denmark.

(received on ??.??.???)

The paper describes a systematic method for the tailoring of dispersion properties of slab-based photonic crystal waveguides. The method is based on the topology optimization method which consists in repeated finite element frequency domain analyses, analytical sensitivity analyses and gradient based design updates. The goal of the optimization process is to come up with slow light, zero group velocity dispersion photonic waveguides or photonic waveguides with tailored dispersion properties for dispersion compensation purposes. Two examples concerning reproduction of a specific dispersion curve and design of a wide bandwidth, constant low group velocity waveguide demonstrate the efficiency of the method.

Keywords: Dispersion engineering, group velocity, photonic crystals, topology optimizaton. 2000 Mathematics Subject Classifications: 81V99, 49Q10. 


\section{Introduction}

Recently there has been a lot of focus on the ability to slow down light for use in optical delay lines, all-optical storage devices, optical buffers, and to increase light-matter interaction for sensing purposes and optical modulation. One way of achieving slow light for these purposes is by use of specifically tailored Photonic Crystal Wave guides (PhCWs) [1-6]. PhCWs are typically made as twodimensional photonic crystal slab structures with line-defect based waveguides. In-plane the light is confined by band gap effects (slow light regime) and index guiding (normal light regime) [6]. The out-of-plane confinement is due to index guiding (dielectric slab surrounded by air). The art of designing PhCWs with specified frequency to wavelength dependency is called dispersion engineering.

For slow light applications the design goals of dispersion engineering are three-fold. The group velocity should be as low as possible, the bandwidth should be maximized [7] and at the same time losses should be minimized which, amongst others, requires that the guided modes should be placed below the light line to ensure confinement in the out-of-plane direction. The goals are conflicting. Extremely low group velocities have been reported [8] but on the cost of extremely small bandwidth. Oppositely, it is no problem to obtain large bandwidth for higher group velocities. A goal closely related to tailoring of the group velocity is to obtain zero Group Velocity Dispersion (GVD). Zero GVD ensures low pulse broadening for transmitted signals. PhCW struc- 
tures for low group velocity and small GVD as well as fairly large bandwidths have been theoretically and experimentally demonstrated $[4,6,9]$. A different goal of dispersion engineering is to design waveguides with specific dispersion properties for dispersion compensation purposes.

Previously suggested dispersion tailored structures have all been designed based on physical arguments and/or trial and error approaches. For example, it has been suggested to narrow down the defect region resulting in so-called W07 PhWGs $[1,4]$, it has been suggested to use a double defect region resulting in W2 PhWGs [9] and it has been suggested to change hole diameters in the two rows of holes closest to the defect in order to control the slow light modes that tend to be less confined in the defect region [6]. As seen, the intuitive approaches have in many cases resulted in very good PhCWs, however, a method that can provide the optimal design for any specified dispersion relation is highly desired. Here, we propose to adapt the topology optimization method for the systematic synthesis of $\mathrm{PhCWs}$ with specified dispersion properties. The topology optimization method was originally developed for mechanical design problems $[10,11]$ but has recently been extended to problems in phononics [12] and PhCWs [13-16]. In our previous works on topology optimization of photonic crystal structures, the main goal was to maximize transmission for waveguide bends, splitters, multiplexors and other basic photonic building blocks. In the present work we reformulate the optimization 
problem in order to minimize the error in obtaining a specified dispersion curve for the guided mode, and at the same time maximizing the mode confinement and avoiding odd and multiple modes in the core region. The topology optimization method is based on a deterministic optimization approach consisting of repeated finite element calculations and design updates using analytically obtained sensitivities and mathematical programming techniques.

The paper is organized as follows. In section 2 we present the $2 \mathrm{D}$ model for a slab-based $\mathrm{PhCW}$ and the associated finite element frequency domain (FEFD) formulation. In section 3 we suggest an optimization strategy. In section 4 we present the analytical sensitivity analysis and discuss the practical implementation of the optimization scheme. In section 5 we present two examples: reproduction of a standard $\mathrm{PhCW}$; and the design of a $\mathrm{PhCW}$ with constant group velocity (and thereby vanishing GVD) and a large bandwidth. Finally, we conclude and discuss possibilities for further work in section 6 .

\section{Modeling}

The governing equation for TE-polarized wave propagation in a twodimensional dielectric domain $\Omega$ is the Helmholtz equation

$$
\nabla \cdot\left(\frac{1}{\varepsilon_{r}(\mathbf{x})} \nabla H\right)+\frac{\omega^{2}}{c^{2}} H=0, \quad \text { for } \boldsymbol{x} \in \Omega
$$


where $c$ is the speed of light in air and $\varepsilon_{r}(\mathbf{x})$ is the space-dependent $x_{1}$-periodic dielectric permittivity. The modeling domain $\Omega$ is a super-cell of dimensions $n \frac{\sqrt{3}}{2} a \times a$, where $a$ is the pitch and $n$ is the number of unit cells perpendicular to the propagation direction (see Figure 1). With wave propagation in the vertical direction (zero horizontal wavevector component), the Floquet-Bloch wave boundary conditions can be written as

$$
\begin{aligned}
H\left(x_{1}, a\right) & =e^{i k a} H\left(x_{1}, 0\right), \quad \text { for } \quad x_{1} \in\left[-n \frac{\sqrt{3}}{4} a, n \frac{\sqrt{3}}{4} a\right], \\
H\left(-n \frac{\sqrt{3}}{4} a, x_{2}\right) & =H\left(n \frac{\sqrt{3}}{4} a, x_{2}\right), \quad \text { for } \quad x_{2} \in\left[-\frac{a}{2}, \frac{a}{2}\right],
\end{aligned}
$$

where $k$ is the wavenumber. In finite element notation, the Helmholtz equation (1) together with the boundary conditions (2) and (3) lead to the Hermitian eigenvalue problem

$$
\left(\mathbf{K}_{k}-\omega^{2} \mathbf{M}\right) \mathbf{U}=\mathbf{0}
$$

where the boundary conditions are imposed by a penalty approach, $\mathbf{K}$ is the global finite element matrix originating from the divergence term of the Helmholtz equation (1), $\mathbf{M}$ is the global finite element matrix originating from the second term of the Helmholtz equation (1) and subscript $k$ means dependence of the wavenumber $k$. To cover all wavelengths, the eigenvalue problem must be solved for wavenumbers in the interval $k \in[0,0.5]$.

The result of the finite element eigenvalue analysis for a typical $\mathrm{PhCW}$ 
structure (Figure 1) consisting of $n=12$ unit cells including a central defect, a so-called W1 waveguide, is shown in Figure 2a. In the computations we used $\varepsilon_{r}=12.08\left(n_{S i} \approx 3.5\right)$ and hole diameter $d=0.6 a$. The structure is discretized using 4080 quadrilateral 4-node elements (20 by 17 elements per unit cell). Although this discretization is rather coarse from a convergence point of view, it provides fairly accurate results and of primary concern quicker computational time for the optimization process. It is our experience that finer discretizations do not result in significantly different topological designs (see section 4 for further discussions on this topic). In Figure 2a) the slab mode regions are indicated with grey and the even and odd guided modes are indicated with solid and dashed lines, respectively. The dash-dotted line indicates the light line over which light will not be confined in the slab for the out-of-plane direction.

The group velocity $v_{g}$ of a guided mode is defined as

$$
v_{g}=\frac{\partial \omega}{\partial k}=\frac{c}{n_{g}}
$$

i.e., the slope of the dispersion curve. Here $n_{g}$ is the group index. Likewise, the Group Velocity Dispersion (GVD) coefficient $\beta_{2}$ is defined as

$$
\beta_{2}=\frac{\partial^{2} k}{\partial \omega^{2}}=\frac{\partial n_{g}}{\partial \omega} \frac{1}{c}
$$

Studying the band diagram in Figure 2a) and the group velocity and group 


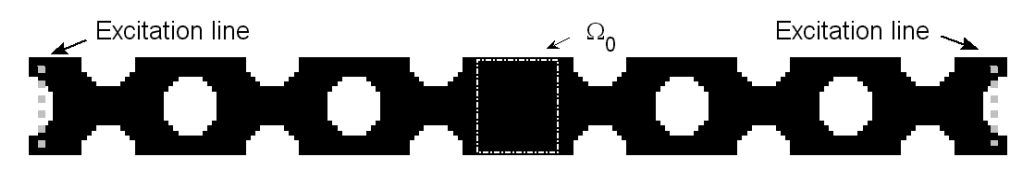

Figure 1. Supercell composed of $n=12$ unit cells including the central defect region $\Omega_{0}$. Dotted vertical lines indicate the line excitations.

index plots for the even guided mode in Figure $2 b$ ) and c), we note that the group velocity is large and close to the group velocity in pure dielectric $\left(v_{g}=\right.$ $1 / n_{S i}=1 / 3.5 \approx 0.29, n_{S i}=3.5$ ) for above the light line (for $k<0.3$ ). This indicates that the guided mode is index guided in this wavenumber interval. Oppositely, the dispersion curve flattens out, i.e. the group velocity is small and the group index high below the light line. This indicates that the guided mode is band gap guided since the wave must be interacting with the holes surrounding the defect [6].

Although the band diagram and the associated group velocity and index plots in Figure 2 offer much insight into the properties of a $\mathrm{PhCW}$, the next section will show that the underlying eigenfrequency problem (4) is not well suited for the optimization problem formulation. Instead we propose to model the waveguide by harmonically varying even and odd excitations and to evaluate the dispersion behavior by confinement measures. Based on this idea, the new finite element problems to be solved are

$$
\left(\mathbf{K}_{k}+i \omega \mathbf{C}-\omega^{2} \mathbf{M}\right) \mathbf{U}=\mathbf{S}_{k, \omega} \mathbf{U}=\mathbf{F}^{e v e n}
$$


a)

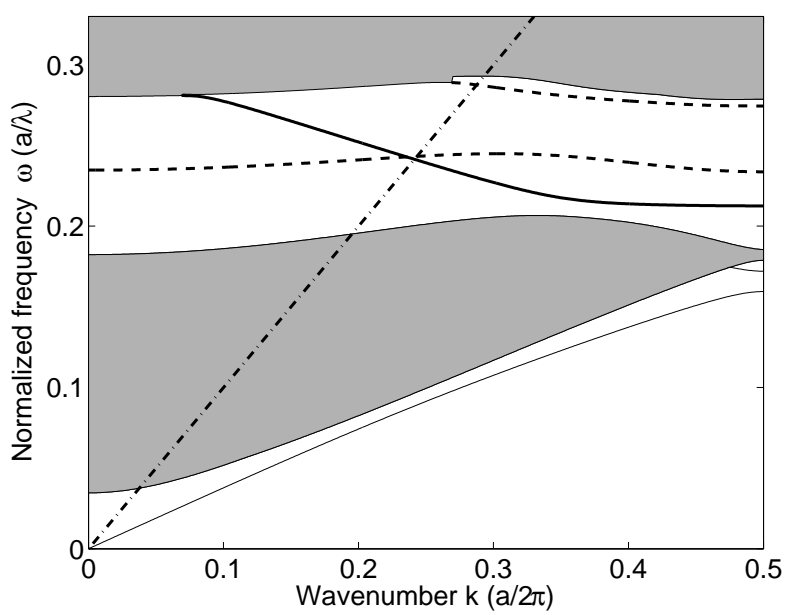

b)
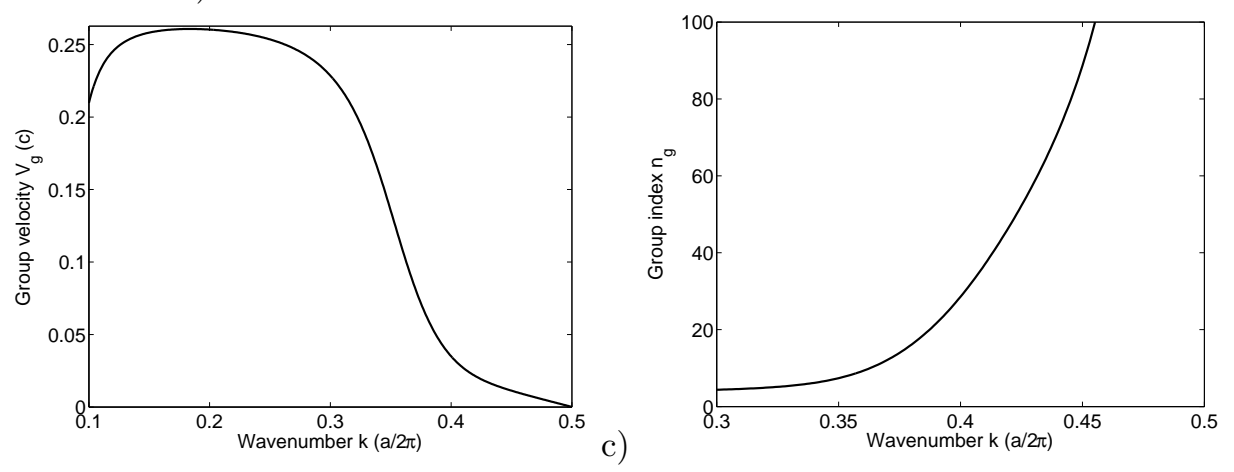

Figure 2. a) Band diagram for the supercell in Figure 1. The bold solid line indicates the even guided mode, the bold dashed lines indicate odd guided modes and the dash-dotted line is the light line. b) Group velocity of the even guided mode. c) Group index of the even guided mode.

and

$$
\left(\mathbf{K}_{k}+i \omega \mathbf{C}-\omega^{2} \mathbf{M}\right) \mathbf{V}=\mathbf{S}_{k, \omega} \mathbf{V}=\mathbf{F}^{o d d}
$$

where $\mathbf{C}$ is a damping matrix (introduced for stabilization of the optimization scheme), $\mathbf{S}_{k, \omega}$ is the frequency and wavenumber dependent finite element system matrix and $\mathbf{F}^{\text {even }}$ and $\mathbf{F}^{\text {odd }}$ are even and odd loading vectors. The 
boundary conditions are the same as before (given by (2) and (3)). For the even excitation case, the load $\mathbf{F}^{\text {even }}$ consists of two symmetrically placed vertical line sources at the outer parts of the supercell (see Figure 1) which excite even modes. In the odd excitation case $\mathbf{F}^{\text {odd }}$ the line sources are out of phase with each other in order to excite odd modes. To measure the confinement of the excited waves to the center region $\Omega_{0}$ we define an even mode confinement measure

$$
I^{\text {even }}\left(H_{k, \omega}^{e v e n}\right)=\frac{\int_{\Omega_{0}}\left|H_{k, \omega}^{e v e n}\right|^{2} d \Omega}{\int_{\Omega}\left|H_{k, \omega}^{e v e n}\right|^{2} d \Omega} .
$$

Likewise, the confinement measure for odd modes is defined as

$$
I^{\text {odd }}\left(H_{k, \omega}^{\text {odd }}\right)=\frac{\int_{\Omega_{0}}\left|\operatorname{sign}\left(x_{1}\right) H_{k, \omega}^{\text {odd }}\right|^{2} d \Omega}{\int_{\Omega}\left|H_{k, \omega}^{o d d}\right|^{2} d \Omega},
$$

In discrete form these measures can be written as

$$
I^{\text {even }}(\mathbf{U})=\frac{\overline{\mathbf{U}}^{T} \mathbf{L} \mathbf{U}}{\overline{\mathbf{U}}^{T} \mathbf{U}}
$$

and

$$
I^{\text {odd }}(\mathbf{V})=\frac{\overline{\mathbf{V}}^{T} \mathbf{A} \mathbf{V}}{\overline{\mathbf{V}}^{T} \mathbf{V}},
$$

where overbar means complex conjugate, $\mathbf{L}$ is a diagonal matrix with ones in the degrees of freedom corresponding to nodal points lying within the core region $\Omega_{0}$, $\mathbf{A}$ is a diagonal matrix with ones in the degrees of freedom corre- 

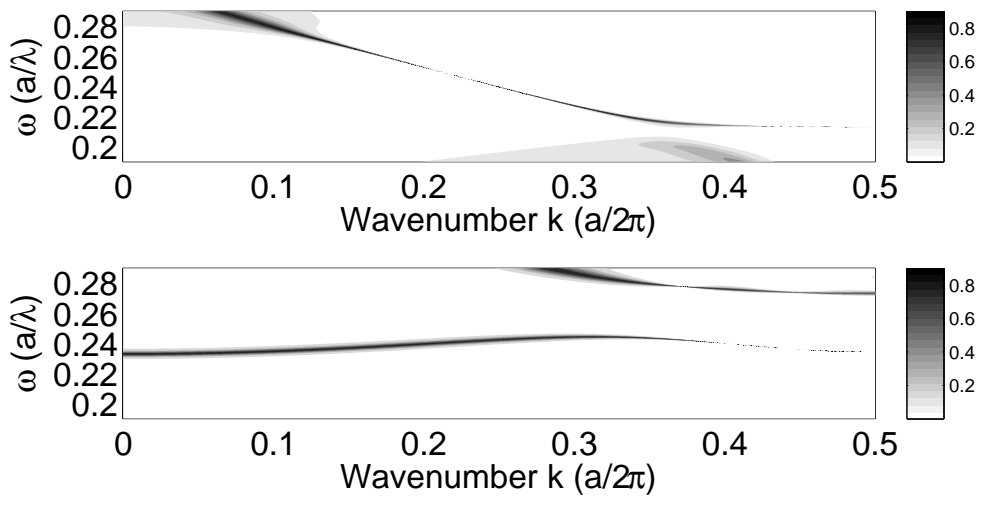

Figure 3. Confinement plots for even (top) and odd excitation (bottom) for the supercell in Figure 1.

sponding to nodal points lying within the left half of the core region $\Omega_{0}$ and minus ones in the right half and $\mathbf{U}$ and $\mathbf{V}$ are the solution vectors to the even and odd excitation problems defined by equations (7) and (8).

Mappings for the even and odd confinement measures for the frequency wavenumber region $\omega \in[0.19,0.29], k \in[0,0.5]$ are shown in Figure 3. It is clearly seen how the confinements are high in $(k, \omega)$ points corresponding to the even and odd guided modes from Figure 2a), but also non-guided modes give rise to non-zero intensities in the slab-guided regions.

\section{Optimization problem}

The goal of the optimization is to make the guided mode follow a specified dispersion curve defined by $\omega^{*}\left(k^{*}\right)$ for wavevectors in the interval $k^{*} \in[\underline{k}, \bar{k}]$. Since we in practice only evaluate $\omega^{*}$ for discrete wavenumbers $k_{i}^{*}$ a practical 
goal of the optimization would be to minimize the errors between the actual and the specified dispersion curves for $m$ discrete wavenumber-frequency pairs $\left(k_{i}^{*}, \omega_{i}^{*}\right)$, i.e.

$$
\min : \Phi=\sum_{i=1}^{m}\left(\omega\left(k_{i}^{*}\right)-\omega_{i}^{*}\right)^{2},
$$

where $k_{i}^{*}$ are $m$ evenly distributed wavenumbers in the interval $[\underline{k}, \bar{k}]$ and $\omega_{i}^{*}$ are the corresponding prescribed eigenvalues. In practice, one can use a starting guess with a guided mode whose dispersion curve is close to the prescribed curve and then look for the design that minimizes $\Phi$. In the best case, the mode is still a guided mode after the optimization and if $\Phi$ is minimized to 0 it has the specified dispersion. However, in the worst case (which is more likely) the dispersion curve is equal to the decided (i.e. $\Phi \approx 0$ ) but the mode is no longer guided, or in other words, it is no longer confined to the center region $\Omega_{0}$ of the super cell. Therefore, we need to define an optimization goal which not only results in the wanted dispersion curve but also ensures confinement to the center region $\Omega_{0}$. A new objective function fulfilling these criteria is

$$
\max : \Psi=\frac{1}{m} \sum_{i=1}^{m} I_{i}^{e v e n},
$$

where $I_{i}^{\text {even }}$ corresponds to the confinement measure (defined in (9) and (11)) evaluated for the $m$ prescribed wavenumber-frequency pairs $\left(k_{i}^{*}, \omega_{i}^{*}\right)$. In the ideal situation where $\Psi=1$ after optimization, we have that all the energy in 
the guided mode is confined to the center region $\Omega_{0}$ and that we have obtained the wanted dispersion curve. If $\Psi<1$ it means that some of the mode is not fully confined to the center region (which is usually the case for slow light) or that some of the energy is taken up by other (higher order) guided modes. However, higher order even modes are not favored by the objective function $\Psi$ since their energy is not confined to the center region only and therefore we do not expect these to create problems. For the odd modes, we introduce extra constraints to force them away from the center region. The odd mode constraints are defined as

$$
g_{i}=I_{i}^{\text {odd }} \leq \delta, i=1, \ldots, m,
$$

where $\delta$ is a small number and the odd confinement measure is defined by (10) and (12). A small value of $g$ ensures that no odd modes can be near the prescribed even modes.

Now we have defined the goals of the optimization problem and what is missing is to define the design parametrization, i.e. the design variables of the optimization problem. The basic idea of the topology optimization method is to allow the relative material density $\rho_{e} \in[0,1]$ in each element to be a design variable. The discrete values $\rho_{e}=0$ and $\rho_{e}=1$ correspond to elements with pure air or pure dielectric, respectively. Intermediate values are allowed during the design process in order to be able to use gradient based optimization tools. 
Despite of the continuous design variables, however, it is our experience that optimized designs always are close to being discrete. The reason being that wave guiding intrinsically requires maximum contrast. The inverse dielectric constant in each element depends on the element relative density in a simple linear fashion

$$
\frac{1}{\varepsilon_{r}\left(\rho_{e}\right)}=1+\rho_{e}\left(\frac{1}{\varepsilon_{r d}}-1\right)
$$

where $\varepsilon_{r d}$ is the permittivity of the dielectric (i.e. $\varepsilon_{r d}=\varepsilon_{S i}$ ).

With these definitions, the final optimization problem can be defined as

$$
\left.\begin{array}{rl}
\max _{\boldsymbol{\rho}} & : \Psi(\boldsymbol{\rho})=\frac{1}{m} \sum_{i=1}^{m} I_{i}^{\text {even }}\left(\boldsymbol{U}_{i}(\boldsymbol{\rho})\right) \\
\text { s.t. }: & g_{i}(\boldsymbol{\rho})=I_{i}^{\text {odd }}\left(\mathbf{V}_{i}(\boldsymbol{\rho})\right) \leq \delta, \quad i=1, \ldots, m, \\
\quad: \mathbf{0} \leq \boldsymbol{\rho} \leq \mathbf{1},
\end{array}\right\}
$$

where $\boldsymbol{\rho}$ is an $N$-vector containing the $N$ design variables (relative element densities). In order to get better resolution for small confinement values, we actually maximize $\log \left(I_{i}^{\text {even }}\right)$ instead of maximizing $I_{i}^{\text {even }}$ and likewise the constraints are rewritten as $\log \left(I_{i}^{\text {odd }}\right) \leq \log (\delta)$ instead of $I_{i}^{\text {odd }} \leq \delta$. In (17) $\boldsymbol{U}_{i}(\boldsymbol{\rho})$ and $\boldsymbol{V}_{i}(\boldsymbol{\rho})$ are the solutions to the finite element problems

$$
\begin{aligned}
& \boldsymbol{S}_{k_{i}, \omega_{i}}(\boldsymbol{\rho}) \boldsymbol{U}_{i}=\boldsymbol{F}^{\text {even }}, \quad i=1, \ldots, m, \\
& \boldsymbol{S}_{k_{i}, \omega_{i}}(\boldsymbol{\rho}) \boldsymbol{V}_{i}=\boldsymbol{F}^{\text {odd }}, \quad i=1, \ldots, m .
\end{aligned}
$$




\section{Sensitivity analysis and implementation}

The optimization problem defined in the previous section is solved using a mathematical programming tool, the Method of Moving Asymptotes (MMA) [17]. It is a deterministic gradient based method that improves on a given initial design distribution following an iterative procedure. For each iteration the objective function and the constraints as well as sensitivities with respect to the design variables $\boldsymbol{\rho}$ must be evaluated. Based on this information the MMA yields an improved design and the process continues. The iterations are stopped when the changes in design variables become insignificant. More details about this standard approach to topology optimization are found in [11].

Efficient sensitivity computations are essential for the success of a gradient based optimization scheme. For the kind of problem considered, the sensitivity analyses can be performed analytically with insignificant computational costs. Based on the adjoint method for sensitivity analysis and using that the load vector is independent on design, the sensitivity of the objective function $\Psi$ (14) with respect to a change in design variable $\rho_{e}$ can be found as

$$
\frac{\partial \Psi}{\partial \rho_{e}}=\sum_{i=1}^{m} \operatorname{Re}\left(\boldsymbol{\lambda}_{i}^{T} \frac{\partial \boldsymbol{S}_{k_{i}, \omega_{i}}(\boldsymbol{\rho})}{\partial \rho_{e}} \boldsymbol{U}_{i}\right)
$$


where $\boldsymbol{\lambda}_{i}$ are the solutions to the $m$ adjoint problems

$$
\boldsymbol{S}_{k_{i}, \omega_{i}}^{T} \overline{\boldsymbol{\lambda}}_{i}=\frac{2}{\overline{\boldsymbol{U}}_{i}^{T} \boldsymbol{U}_{i} I_{i}^{\text {even }}}\left(\boldsymbol{L}-I_{i}^{\text {even }} \cdot \boldsymbol{I}\right) \boldsymbol{U}_{i}
$$

Here $\boldsymbol{I}$ stands for the identity matrix. The design dependency of the finite element matrix $\boldsymbol{S}_{k_{i}, \omega_{i}}$ comes from the design-weighted assembled stiffness matrix

$$
\boldsymbol{K}=\sum_{e=1}^{N} \frac{1}{\varepsilon_{r}\left(\rho_{e}\right)} \boldsymbol{K}_{e}
$$

hence

$$
\frac{\partial \boldsymbol{S}_{k_{i}, \omega_{i}}(\boldsymbol{\rho})}{\partial \rho_{e}}=\frac{1}{\varepsilon_{r d}} \boldsymbol{K}_{e}
$$

where $\mathbf{K}_{e}$ is the element matrix for an air-filled element $\left(\varepsilon_{r}=1\right)$. The sensitivities of the constraints $g_{i}$ can similarly be computed as

$$
\frac{\partial g_{i}}{\partial \rho_{e}}=\operatorname{Re}\left(\boldsymbol{\mu}_{i}^{T} \frac{\partial \boldsymbol{S}_{k_{i}, \omega_{i}}(\boldsymbol{\rho})}{\partial \rho_{e}} \boldsymbol{V}_{i}\right)
$$

where $\boldsymbol{\mu}_{i}$ are the solutions to the adjoint problems from (20) with $\mathbf{U}_{i}$ substituted by $\mathbf{V}_{i}$ and $\boldsymbol{\lambda}_{i}$ substituted by $\boldsymbol{\mu}_{i}$. The adjoint problems in (20) are very cheap to solve since the factorizations of the system matrices $\boldsymbol{S}_{k_{i}, \omega_{i}}$ already are available from the direct analyses (18). Therefore the sensitivity analysis can be performed at virtually no added cost compared to the solution of the original finite element problem.

Solving the undamped harmonic problems (18) creates solutions with very 
sharp resonance peaks as seen in Figure 3. This means that small design changes may cause big shifts in response and thereby creating trouble for the optimization algorithm. In order to relieve this problem we use a continuation approach (previously suggested in [15]) based on an artificial damping term as given by the damping matrix $\boldsymbol{C}$ in (7) and (8). In the beginning of the optimization a fairly high artificial damping of $C=0.05 \mathrm{M}$ provides for smoother and broader peaks. Later, as the design has been allowed to converge, the damping is gradually diminished in order to model and optimize the real undamped response.

As mentioned earlier, it is our experience that the design problem converges to almost discrete solutions, i.e. almost all optimized design variables take the discrete values 0 or 1 , even though the problem is described with continuous variables. A problem, however, is that optimized design may exhibit very fine details, i.e. material variations on the element scale. These details are not desired, neither from a modeling point of view, nor from the manufacturing point of view. Different regularization techniques were reviewed in [18] but here we make use of a discrete feature size control scheme suggested in [19]. The regularizing filter technique is based on the morphological image operator close and does not allow holes below a certain radius $r_{\text {min }}$ to appear in the optimized topologies. 


\section{Examples}

We have used the suggested algorithm to design two waveguides with prescribed dispersion properties. For both examples we discretized the supercell consisting of $n=12$ unit cell with 4080 quadrilateral 4-node finite elements. Experiments with a coarser discretizations yielded similar results, but with coarser boundaries due to the pixel-like discretization. From this study and previous experience, we conclude that a finer discretization will not change the results significantly and only increase computational costs. We used a design domain consisting of the inner 7 unit cells for both examples, since the outer rows of holes hardly show any interaction with the confined light in the waveguide core region $\Omega_{0}$. The optimization procedure as well as the associated finite element solver is implemented in Matlab. Since this high-level programming language is not well suited for finite element analysis, computational times are rather slow. Typically we use 1000-1500 iterations for convergence, each taking 20s, i.e. a full optimization process may take up to 8 hours on a standard PC. In the future we plan to code the scheme in a lower level, but speedier, programming language and expect significant time savings. For the optimization problem (17), the bound for the odd confinements are set to $\delta=0.05$, but it should be mentioned, that these constraints never are active for the optimized designs. 


\subsection{Reconstruction of the original guided mode}

As the first example we want to prove that the optimization scheme can reproduce the dispersion behavior of the perfect periodic crystal with a central defect from Figure 1, without initial assumption of periodicity. Therefore we choose five $\left(\omega_{i}^{*}, k_{i}^{*}\right)$-points from the dispersion curve of the even guided mode in Figure 2a) as the goal of the optimization problem. For this first example we do not make use of the regularization technique discussed in section 4 , hence details on the element-size level are allowed in the optimized structure. Figure 4a) shows the initial design of the supercell, i.e. apart from the five outermost cells, the initial design consists of pure dielectric. Figure 4b) shows the band diagram of the initial design as well as the five prescribed points indicated by crosses. It is seen that the prescribed points all are placed in a non-guiding, non band gap slab-region. The optimized design for the $\mathrm{PhCW}$ and the associated dispersion diagram is seen in Figure 5a) and b), respectively. Visually, the dispersion curve is seen to pass almost perfectly through the prescribed points. It is also interesting to see how a band gap region has been formed although no such was present for the initial design in Figure 4. For the reconstructed waveguide we get the following confinement values at the target points: $I_{i}^{\text {even }}=(0.85,0.72,0.59,0.55,0.54),(\Phi=0.65)$ in comparison to $I_{i}^{\text {even }}=(0.89,0.77,0.54,0.47,0.45),(\Phi=0.624)$ for the perfectly periodic design from Figure 1. The numbers indicate that the overall confinement to the 
a)

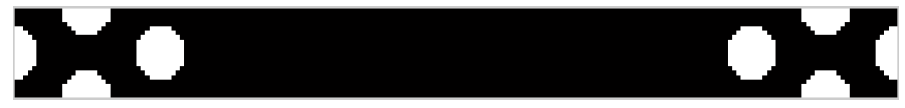

b)

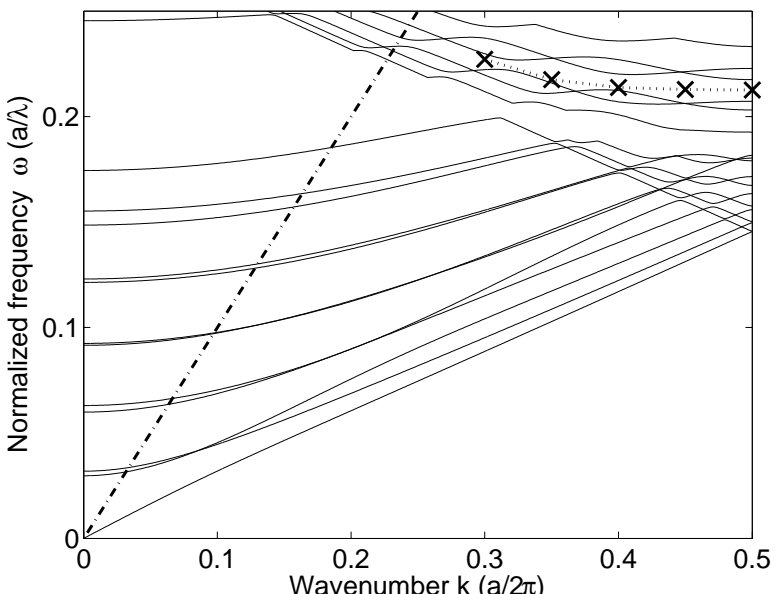

Figure 4. a) Initial design for the reconstruction example. b) Dispersion diagram for the initial design with indicated target points.

a)

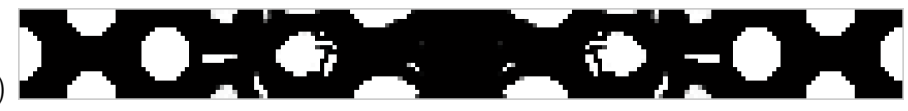

b)

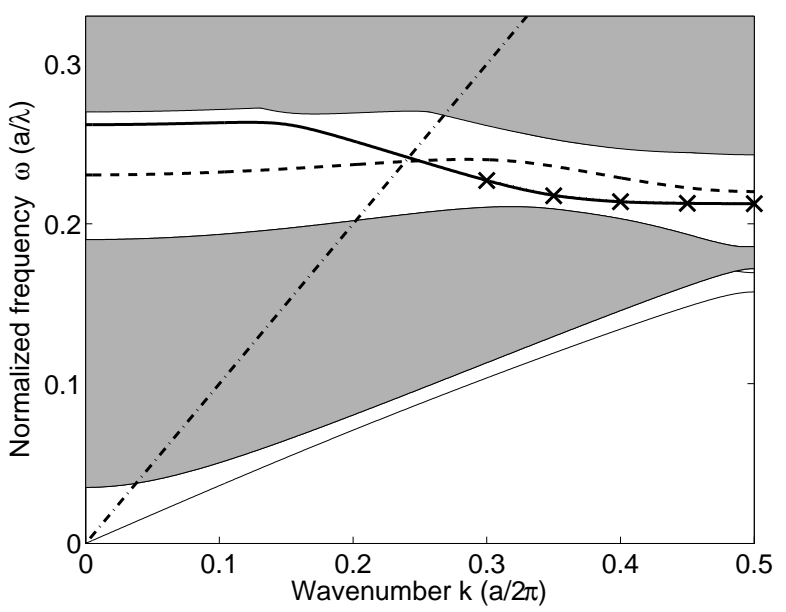

Figure 5. a) Optimized design for the reconstruction example. b) Dispersion diagram for the optimized design. 
central region is better for the optimized design and that this is especially the case for the slow light region $(i=3,4,5)$. It is also noted that there are many small and non-manufacturable details in the optimized topology since the regularization technique was not used. In the next example the regularization filter is employed which makes the resulting topology manufacturable.

\subsection{Design of a PhCW with constant group velocity}

In this example we try to design a $\mathrm{PhCW}$ with constant group velocity $v_{g}=c / 20$ in the wave number interval $k^{*} \in[0.375,0.475]$ (under the light line). To obtain this we choose our target points to lie on a straight line given by $\left\{\left(k_{i}^{*}, \omega_{i}^{*}\right)\right\}=\{(0.375,0.2393),(0.4,0.2383),(0.425,0.2373),(0.45,0.2363)$, $(0.475,0.2353)\}$. As a starting guess we use the perfect periodic waveguide from Figure 1. Moreover, we use a filter method with $r_{\text {min }}=a / 8$ (cf. section 4) to avoid small details in the optimized topology. The optimized $\mathrm{PhCW}$ and the corresponding dispersion diagram are shown in Figure 6a) and b), respectively. The achieved confinement values at the target points are: $I_{i}^{\text {even }}=(0.67,0.57,0.54,0.57,0.63),(\Phi=0.596)$. From the band diagram it seems that the goal of the optimization has been met to perfection. However, studying the group velocity plot in Figure 6c) it is seen that we do obtain an interval with almost constant group velocity $\left(v_{g} \approx c / 20\right.$ for $\left.k \in[0.39,0.43]\right)$, however, this interval is small compared to the target interval. The group in- 
a)
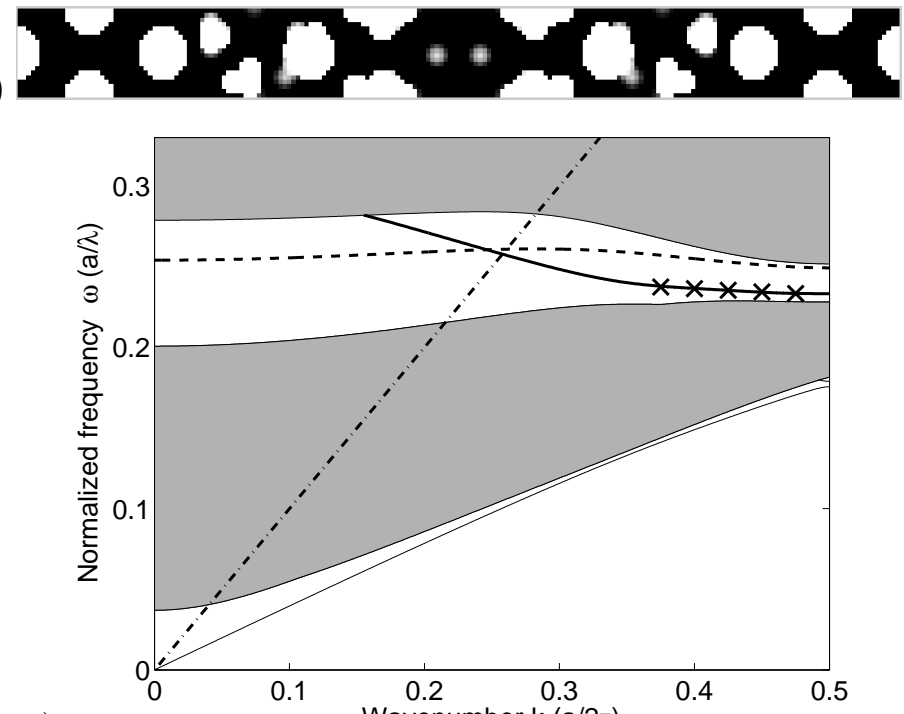

b)

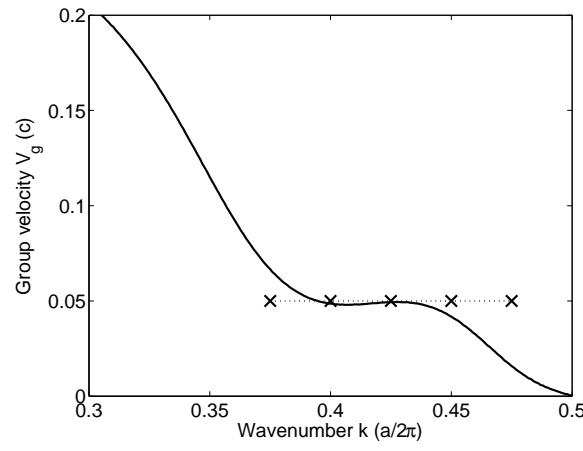

d)

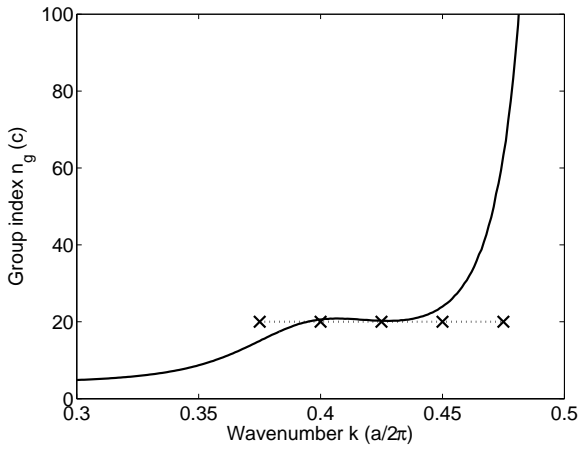

Figure 6. a) Optimized design for constant group velocity example. b) Corresponding band diagram with indication of target points. c) Group velocity. d) Group index.

dex plot in Figure 6d) gives a possible explanation. It is seen that the group index rapidly goes to infinity for $k$ approaching 0.5 . Therefore it is probably impossible to ask for a constant finite group index too close to $k=0.5$. The optimization algorithm finds the best compromise and can therefore not obtain perfect match for the chosen wavenumber interval. TO THE REVIEW- 
ERS: DUE TO THE DEADLINE FOR SUBMISSION TO THIS SPECIAL ISSUE, WE CAN AT PRESENT NOT DEMONSTRATE A BETTER EXAMPLE. HOWEVER, WE HOPE TO IMPROVE THE RESULT FOR THE FINAL VERSION OF THE PAPER WHEN WE HAVE EXPLORED THE SOLUTION SPACE IN MORE DETAIL.

\section{Conclusions}

In this paper we have proposed a systematic tool for dispersion engineering of photonic crystal waveguides. The abilities of the method have been demonstrated on two simple examples. In future work we plan to explore the limits of dispersion engineering in more detail by design for slower light, wider bandwidths, bi-linear dispersion curves and compact dispersion compensating devices. Also we plan to extend the modeling to the full 3D Maxwell equations in order to capture problems with polarization coupling and out-of-plane losses.

\section{Acknowledgements}

This work received support from the Eurohorcs/ESF European Young Investigator Award (EURYI, www.esf.org/euryi) through the grant "Synthesis and topology optimization of optomechanical systems", the New Energy and Industrial Technology Development Organization project (NEDO, Japan), and 
from the Danish Center for Scientific Computing (DCSC).

\section{References}

[1] M. Notomi, K. Yamada, A. Shinya, J. Takahashi, C. Takahashi, and I. Yokohama. Extremely large group-velocity dispersion of line-defect waveguides in photonic crystal slabs. Physical Review Letters, 87(25):253902/1-253902/4, 2001.

[2] S.G. Johnson, P.R. Villeneuve, S. Fan, and J.D. Joannopoulos. Linear waveguides in photoniccrystal slabs. Physical Review B (Condensed Matter), 62(12):8212-8222, 2000.

[3] M. Soljacic, S. G. Johnson, Sh. Fan, M. Ibanescu, E. Ippen, and J.D. Joannopoulos. Photoniccrystal slow-light enhancement of nonlinear phase sensitivity. Journal of the Optical Society of America B: Optical Physics, 19(9):2052-2059, 2002.

[4] A.Y. Petrov and M. Eich. Zero dispersion at small group velocities in photonic crystal waveguides. Applied Physics Letters, 85(21):4866-4868, 2004.

[5] D. Mori and T. Baba. Dispersion-controlled optical group delay device by chirped photonic crystal waveguides. Applied Physics Letters, 85(7):1101-1103, 2004.

[6] L. H. Frandsen, A. V. Lavrinenko, J. Fage-Pedersen, and P. I. Borel. Photonic crystal waveguides with semi-slow light and tailored dispersion properties. Optics Express, 14(20):9444-9450, 2006.

[7] F. Xia, L. Sekaric, and Y. Vlasov. Ultracompact optical buffers on a silicon chip. Nature Photonics, $1: 65-71,2007$.

[8] T. Tanabe, M. Notomi, E. Kuramochi, A. Shinya, and H. Taniyama. Trapping and delaying photons for one nanosecond in an ultrasmall high-q photonic-crystal nanocavity. Nature Photonics, $1: 49-52,2007$.

[9] M.D. Settle, R.J.P. Engelen, M. Salib, A. Michaeli, L. Kuipers, and T.F. Krauss. Flatband slow light in photonic crystals featuring spatial pulse compression and terahertz bandwidth. Optics Express, 15(1):219-226, 2007.

[10] M. P. Bendsøe and N. Kikuchi. Generating optimal topologies in structural design using a homogenization method. Computer Methods in Applied Mechanics and Engineering, 71(2):197$224,1988$.

[11] M. P. Bendsøe and O. Sigmund. Topology Optimization - Theory, Methods, and Applications. 
Springer Verlag, Berlin Heidelberg, 2004.

[12] O. Sigmund and J. S. Jensen. Systematic design of phononic band gap materials and structures by topology optimization. Philosophical Transactions of the Royal Society A: Mathematical, Physical and Engineering Sciences, 361:1001-1019, 2003.

[13] J. S. Jensen and O. Sigmund. Systematic design of photonic crystal structures using topology optimization: Low-loss waveguide bends. Applied Physics Letters, 84(12):2022-2024, 2004.

[14] P. I. Borel, A. Harpøth, L. H. Frandsen, M. Kristensen, J. S. Jensen P. Shi, and O. Sigmund. Topology optimization and fabrication of photonic crystal structures. Optics Express, 12(9):19962001, 2004. http: //www. opticsexpress.org/abstract.cfm?URI=OPEX-12-9-1996.

[15] J. S. Jensen and O. Sigmund. Topology optimization of photonic crystal structures: A highbandwidth low-loss T-junction waveguide. Journal of the Optical Society of America B: Optical Physics, 22(6):1191-1198, 2005.

[16] P. I. Borel, L. H. Frandsen, A. Harpøth, M. Kristensen, J. S. Jensen, and O. Sigmund. Topology optimised broadband photonic crystal Y-splitter. Electronics Letters, 41(2):69-71, 2005.

[17] K. Svanberg. The Method of Moving Asymptotes - A new method for structural optimization. International Journal for Numerical Methods in Engineering, 24:359-373, 1987.

[18] O. Sigmund and J. Petersson. Numerical instabilities in topology optimization: A survey on procedures dealing with checkerboards, mesh-dependencies and local minima. Structural Optimization, 16(1):68-75, 1998.

[19] O. Sigmund. Morphology-based black and white filters for topology optimization. Structural and Multidisciplinary Optimization, 2007. in press. 